Provided for non-commercial research and education use. Not for reproduction, distribution or commercial use.

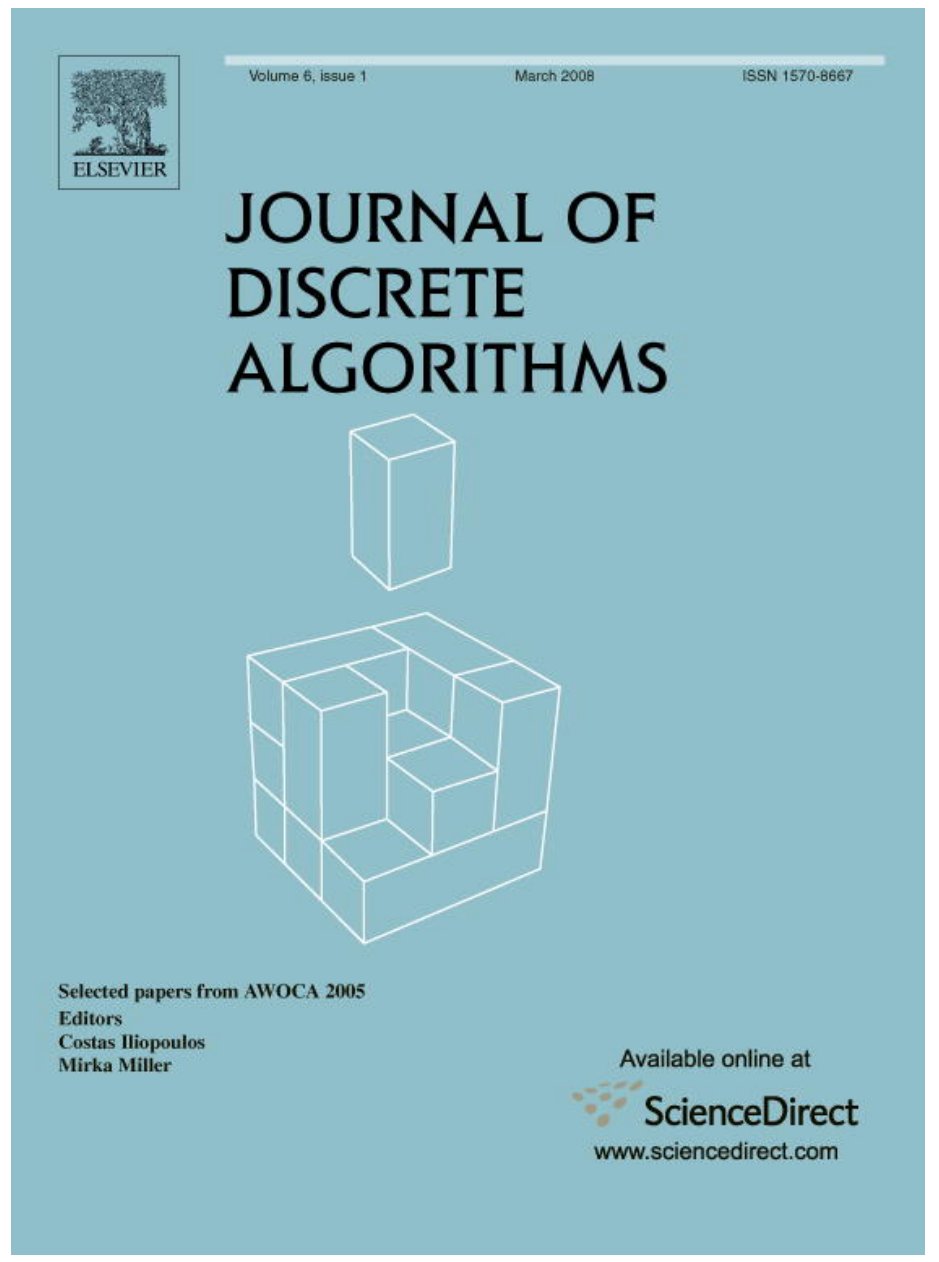

This article was published in an Elsevier journal. The attached copy

is furnished to the author for non-commercial research and education use, including for instruction at the author's institution, sharing with colleagues and providing to institution administration.

Other uses, including reproduction and distribution, or selling or licensing copies, or posting to personal, institutional or third party websites are prohibited.

In most cases authors are permitted to post their version of the article (e.g. in Word or Tex form) to their personal website or institutional repository. Authors requiring further information regarding Elsevier's archiving and manuscript policies are encouraged to visit:

http://www.elsevier.com/copyright 


\title{
On-line scheduling of parallel jobs on two machines
}

\author{
Wun-Tat Chan ${ }^{\mathrm{a}, *, 1}$, Francis Y.L. Chin ${ }^{\mathrm{a}, 2}$, Deshi Ye $^{\mathrm{a}}$, Guochuan Zhang ${ }^{\mathrm{b}, 3}$, Yong Zhang ${ }^{\mathrm{a}}$ \\ a Department of Computer Science, The University of Hong Kong, Pokfulam Road, Hong Kong \\ b Department of Mathematics, Zhejiang University, Hangzhou 310027, China
}

Received 14 November 2005; accepted 16 July 2006

Available online 25 October 2006

\begin{abstract}
We study the problem of on-line scheduling of parallel jobs on two machines. The jobs are parallel in the sense that each of them specifies the number of processors, in this case 1 or 2, required for simultaneous processing. The jobs are presented one by one. Upon receiving a job, we must assign the job to a time slot in the schedule before the next job is presented. No re-assignment is allowed. The goal is to minimize the makespan of the final schedule. There is a straightforward algorithm which achieves a competitive ratio of 2 . In this paper we show that no on-line algorithm can have a competitive ratio less than $1+\sqrt{2 / 3}(\approx 1.816)$. We also study two special cases of the problem: (i) Jobs arrive in a non-decreasing order of processing times where we give an optimal algorithm with competitive ratio $3 / 2$; (ii) Jobs arrive in a non-increasing order of processing times where we show that no on-line algorithm has a competitive ratio less than $9 / 7$ and give a greedy algorithm with a competitive ratio $4 / 3$.
\end{abstract}

(c) 2006 Elsevier B.V. All rights reserved.

Keywords: Jobs scheduling; Online algorithms; Competitive Analysis

\section{Introduction}

In the classical job scheduling, each job requires only a single machine (or processor) for processing. However, this assumption may not apply to modern computer systems, especially in the parallel supercomputers, where some jobs can only be processed on several processors in parallel. The scheduling model for parallel jobs has been proposed and studied extensively [1-7] in recent years. The problem we study in this paper can be described as follows. A job $j=(s, p)$ is associated with two parameters $p$ and $s$, where $p$ is the processing time of the job and $s$ is the number of machines required for simultaneous processing. The jobs are presented one by one. The problem is on-line in the sense that upon receiving a job and before the next job is presented, we need to assign the job to a time slot of the schedule so that the required number of machines is available. No splitting of jobs (i.e., preemption) and no re-assignment is

\footnotetext{
A preliminary version of the paper appeared in Proceedings of the Sixteenth Australasian Workshop on Combinatorial Algorithms.

* Corresponding author.

E-mail addresses: wtchan@cs.hku.hk (W.-T. Chan), chin@cs.hku.hk (F.Y.L. Chin), yedeshi@cs.hku.hk (D. Ye), zgc@ zju.edu.cn (G. Zhang), yzhang@cs.hku.hk (Y. Zhang).

1 The research was supported in part by Hong Kong RGC Grants HKU5172/03E.

2 The research was supported in part by Hong Kong RGC Grants HKU7142/03E.

3 The research was supported in part by NSFC (10231060).
} 
allowed. The goal is to minimize the makespan of the final schedule, i.e., the completion time of the last job to finish in the schedule. In this paper we focus on the case of two machines and therefore each job can request for either 1 or 2 machines for processing, which is denoted as the 1-machine job or 2-machine job, respectively.

We use the competitive analysis [8] to measure the performance of an on-line algorithm. For any input job sequence $I$, let $C_{A}(I)$ denote the makespan of the schedule produced by the on-line algorithm $A$ and $C_{O P T}(I)$ denote the makespan of the optimal schedule. We say that $A$ is $k$-competitive if

$$
\sup _{I}\left\{\frac{C_{A}(I)}{C_{O P T}(I)}\right\} \leqslant k
$$

We also say that $k$ is the competitive ratio of $A$. Notice that for any input job sequence, a $k$-competitive on-line algorithm can always give a schedule with makespan at most $k$ times that of the optimal schedule. For simplicity, we use $C_{A}$ and $C_{O P T}$ instead of $C_{A}(I)$ and $C_{O P T}(I)$ if there is no confusion.

If in our problem all jobs require one machine only, it is the traditional job scheduling problem in multiple machines, first investigated by Graham [9]. A fundamental algorithm called the List Scheduling algorithm [9] is to schedule a newly arrived job to a machine in which the job can start as early as possible. By Graham's analysis, List Scheduling algorithm can be shown to be $(2-1 / m)$-competitive, where $m$ is the number of machines. Faigle et al. [10] showed that the List Scheduling algorithm is in fact an optimal algorithm for 2 and 3 machines.

In general, if a job can request any number of available machines, Johannes presented an on-line algorithm with competitive ratio 12 [7]. She also proved that no on-line algorithm is better than 2.25-competitive [7]. Ye and Zhang improved the upper bound by giving an 8-competitive on-line algorithm [6], which was then further improved by Ye with a 7-competitive on-line algorithm [11]. There were also some results for the cases where some extra information on the jobs is known in advance. If the jobs arrive in a non-increasing order of processing times, Ye and Zhang gave an on-line algorithm with competitive ratio 2 [6]. If the longest processing time is known, Ye gave an on-line algorithm with competitive ratio 4 [11].

In this paper we investigate the two machines case in scheduling parallel jobs. We prove a lower bound of $1+\sqrt{2 / 3}$ on the competitive ratio of any on-line algorithm. We also study two special cases of the problem and give a constant competitive on-line algorithm for each of the cases. (i) When the jobs arrive in a non-decreasing order of processing times, we give an optimal algorithm which is (3/2)-competitive. (ii) When the jobs arrive in a non-increasing order of processing times, we show that no on-line algorithm has a competitive ratio less than $9 / 7$ and we give a (4/3)competitive on-line algorithm.

The rest of the paper is organized as follows. In Section 2 we give an adversary to show the lower bound of $1+\sqrt{2 / 3}$. In Section 3 we present a greedy on-line algorithm. For each of two special case inputs where the jobs arrive in either non-decreasing or non-increasing order of the processing times, we show that the greedy algorithm is constant competitive. The greedy algorithm is indeed optimal in the first case.

\section{Lower bound}

In this section we show that for scheduling parallel jobs on two machines, no on-line algorithm can achieve a competitive ratio less than $1+\sqrt{2 / 3}(\approx 1.816)$. Before we prove the lower bound, we point out that, on the other hand, there is a straightforward on-line algorithm that can guarantee a competitive ratio of 2 . The algorithm simply schedules all jobs one after the other leaving no idle time between jobs. For the 1-machine jobs, they are all scheduled to one particular machine only. In the worst case, there is no 2-machine job. The optimal schedule has all jobs evenly scheduled in the two machines but it still requires a makespan at least half the makespan of the on-line schedule, which is equal to the total processing time.

We prove the lower bound on the competitive ratios of all on-line algorithms by giving an adversary job sequence such that any on-line algorithm ALG has a competitive ratio at least $1+\sqrt{2 / 3}$. The adversary job sequence $S$ consists of at most 5 jobs, $j_{1}, j_{2}, j_{3}, j_{4}$, and $j_{5}$ arriving in that order. The adversary runs as follows. For $i=1$ to 5 , after ALG schedules $j_{i}$, if the ratio of the makespans of the on-line schedule and the optimal schedule for jobs $j_{1}, \ldots, j_{i}$ is at least $1+\sqrt{2 / 3}$, then the adversary stops.

We let $j_{1}=(1,1)$ where the first ' 1 ' denotes the required number of machines and the next ' 1 ' denotes the required processing time. The parameters for the subsequent jobs depend on how the on-line algorithm schedules the previous jobs. Without loss of generality, suppose an on-line algorithm schedules $j_{1}$ in Machine 1 at some time $h_{1} \geqslant 0$ (see 


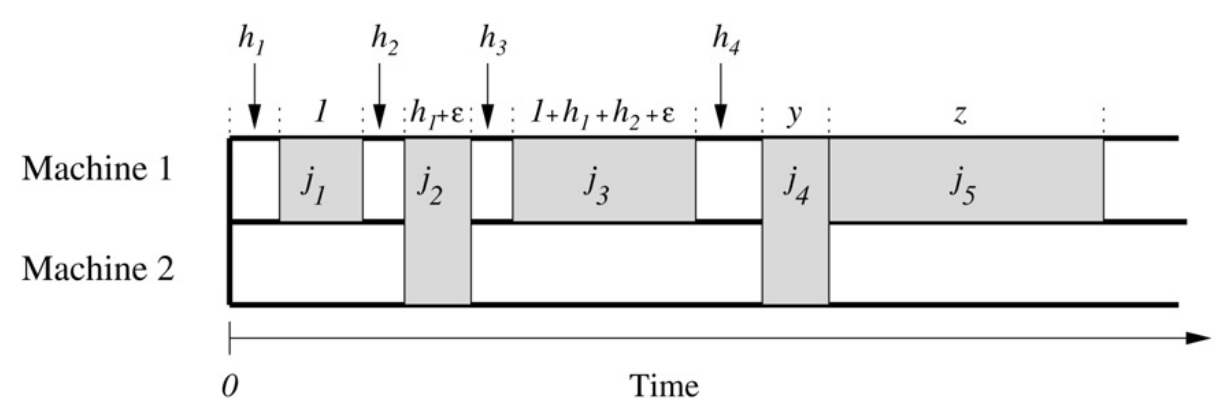

Fig. 1. An on-line schedule for the adversary job sequence $S$.

Fig. 1). We let $j_{2}=\left(2, h_{1}+\epsilon\right)$ where $\epsilon$ is a sufficiently small positive constant (the exactly value of $\epsilon$ will be known in the proof of the lower bound). Since the processing time of $j_{2}$ is larger than $h_{1}$ (the available slot before $j_{1}$ ), $j_{2}$ must be scheduled after $j_{1}$. Suppose $j_{2}$ is scheduled $h_{2}$ units after $j_{1}$. Next, let $j_{3}=\left(1,1+h_{1}+h_{2}+\epsilon\right)$. Similarly, since $j_{3}$ cannot fit into any available slot in either machine before $j_{2}, j_{3}$ must be scheduled after $j_{2}$. Suppose $j_{3}$ is scheduled $h_{3}$ units after $j_{2}$. Since it is indifferent to schedule $j_{3}$ in either machine after $j_{2}$, we assume without loss of generality that $j_{3}$ is scheduled in Machine 1 . Next, let $j_{4}=(2, y)$ where $y=\max \left\{h_{1}, h_{2}, h_{3}\right\}+\epsilon$. Again, since $j_{4}$ cannot fit into any available slot in both machines before $j_{3}$, we assume $j_{4}$ is scheduled $h_{4}$ units after $j_{3}$. Finally, let $j_{5}=(1, z)$ where $z=1+h_{1}+h_{2}+\max \left\{h_{3}+h_{4}+\epsilon, 1\right\}+\epsilon$. We can see that it is also not possible to schedule $j_{5}$ before $j_{4}$. Thus, the best way is to schedule $j_{5}$ immediate after $j_{4}$. It is also indifferent to schedule $j_{5}$ in either machine, so we assume $j_{5}$ is scheduled in Machine 1. See Fig. 1 for the on-line schedule of these jobs.

In the following lemma, we prove that no on-line algorithm can schedule the above adversary job sequence with competitive ratio less than $1+\sqrt{2 / 3}$. Note that the analysis is tight because the on-line algorithm can choose $h_{1}=$ $\sqrt{2 / 3}$ and $h_{2}=h_{3}=h_{4}=0$ and achieve the competitive ratio $1+\sqrt{2 / 3}$ in scheduling the adversary job sequence.

Lemma 1. No on-line algorithm can schedule the adversary job sequence $S$ with competitive ratio less than $1+\sqrt{2 / 3}$.

Proof. Let $S_{i}$ denote the job subsequence $j_{1}, \ldots, j_{i}$. We prove that the maximum among the competitive ratios $\max \left\{C_{A L G}\left(S_{i}\right) / C_{O P T}\left(S_{i}\right) \mid 1 \leqslant i \leqslant 5\right\} \geqslant 1+\sqrt{2 / 3}$. It is shown by the argument that if both $C_{A L G}\left(S_{1}\right) / C_{O P T}\left(S_{1}\right) \leqslant$ $1+\sqrt{2 / 3}-\delta$ and $C_{A L G}\left(S_{3}\right) / C_{O P T}\left(S_{3}\right) \leqslant 1+\sqrt{2 / 3}-\delta$ for some $\delta>0$, then $C_{A L G}\left(S_{5}\right) / C_{O P T}\left(S_{5}\right) \geqslant 1+\sqrt{2 / 3}$.

First, we consider $S_{1}$. The optimal schedule for $S_{1}$ has $j_{1}$ to start at time 0 , so we have

$$
\frac{C_{A L G}\left(S_{1}\right)}{C_{O P T}\left(S_{1}\right)}=1+h_{1} \leqslant 1+\sqrt{\frac{2}{3}}-\delta \Rightarrow h_{1} \leqslant \sqrt{\frac{2}{3}}-\delta .
$$

For the optimal schedule for $S_{3}, j_{1}$ and $j_{3}$ both start at time 0 but on different machines and $j_{2}$ starts at time $1+h_{1}+$ $h_{2}+\epsilon$ on both machines. Therefore, we have

$$
\begin{aligned}
\frac{C_{A L G}\left(S_{3}\right)}{C_{O P T}\left(S_{3}\right)}=\frac{2+3 h_{1}+2 h_{2}+h_{3}+2 \epsilon}{1+2 h_{1}+h_{2}+2 \epsilon} \leqslant 1+\sqrt{\frac{2}{3}}-\delta \\
\Rightarrow h_{3} \leqslant\left(2 \sqrt{\frac{2}{3}}-1-2 \delta\right) h_{1}-\left(1-\sqrt{\frac{2}{3}}+\delta\right) h_{2}-\left(1-\sqrt{\frac{2}{3}}(1+2 \epsilon)+(1+2 \epsilon) \delta\right) \\
\leqslant\left(2 \sqrt{\frac{2}{3}}-1-2 \delta\right) h_{1} \quad \text { let } \epsilon \leqslant \sqrt{\frac{3}{8}}-\frac{1}{2} \\
\leqslant h_{1} .
\end{aligned}
$$

Since $j_{4}=(2, y)$ where $y=\max \left\{h_{1}, h_{2}, h_{3}\right\}+\epsilon$, and $h_{3} \leqslant h_{1}$, we can simplify $y=\max \left\{h_{1}, h_{2}\right\}+\epsilon$.

In the following we consider an offline schedule for $S_{5}$ and analyze the ratio of the makespans of the on-line schedule and this offline schedule. Clearly, this ratio will be at most the competitive ratio of the on-line schedule. The offline schedule has $j_{2}$ to start at time $0, j_{4}$ at time $h_{1}+\epsilon$. Then $j_{1}$ and $j_{5}$ are scheduled at time $h_{1}+y+\epsilon$ in Machine 1 and Machine 2, respectively. Finally, $j_{3}$ is scheduled at time $1+h_{1}+y+\epsilon$ in Machine 1. See Fig. 2 for the offline schedule. 


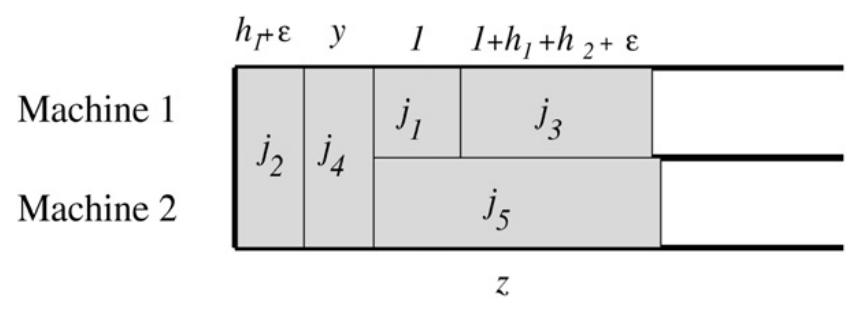

Fig. 2. An offline schedule for the adversary job sequence $S$.

We present the analysis case by case and show that $C_{A L G}\left(S_{5}\right) / C_{O P T}\left(S_{5}\right) \geqslant 1+\sqrt{2 / 3}$.

Case 1. Assume $h_{3}+h_{4}+\epsilon \geqslant 1$. Thus we have $j_{5}=(1, z)$ with $z=1+h_{1}+h_{2}+h_{3}+h_{4}+2 \epsilon$. The competitive ratio is

$$
\begin{aligned}
\frac{C_{A L G}\left(S_{5}\right)}{C_{O P T}\left(S_{5}\right)} & \geqslant \frac{3+4 h_{1}+3 h_{2}+2 h_{3}+2 h_{4}+y+4 \epsilon}{1+2 h_{1}+h_{2}+h_{3}+h_{4}+y+3 \epsilon} \\
& \geqslant \min \left\{2, \frac{1+h_{2}+y+4 \epsilon}{y+3 \epsilon}\right\} \\
& \geqslant \min \left\{2, \frac{h_{1}+h_{2}+\max \left\{h_{1}, h_{2}\right\}+1-\sqrt{2 / 3}+\delta+5 \epsilon}{\max \left\{h_{1}, h_{2}\right\}+4 \epsilon}\right\} \quad \text { as } h_{1} \leqslant \sqrt{\frac{2}{3}}-\delta \\
& =2 \quad \text { let } \epsilon \leqslant \frac{1-\sqrt{2 / 3}+\delta}{3} \\
& \geqslant 1+\sqrt{\frac{2}{3}} .
\end{aligned}
$$

Case 2. Assume $h_{3}+h_{4}+\epsilon<1$. Thus we have $j_{5}=(1, z)$ with $z=2+h_{1}+h_{2}+\epsilon$. The competitive ratio is

$$
\frac{C_{A L G}\left(S_{5}\right)}{C_{O P T}\left(S_{5}\right)}=\frac{4+4 h_{1}+3 h_{2}+h_{3}+h_{4}+y+3 \epsilon}{2+2 h_{1}+h_{2}+y+2 \epsilon} \geqslant \frac{4+4 h_{1}+3 h_{2}+y+3 \epsilon}{2+2 h_{1}+h_{2}+y+2 \epsilon} .
$$

Case 2.1. Assume $h_{1} \leqslant h_{2}$. Thus $y=h_{2}+\epsilon$, and we have

$$
\frac{C_{A L G}\left(S_{5}\right)}{C_{O P T}\left(S_{5}\right)}=\frac{4+4 h_{1}+4 h_{2}+4 \epsilon}{2+2 h_{1}+2 h_{2}+3 \epsilon} \geqslant 1+\sqrt{\frac{2}{3}} \quad \text { let } \epsilon \leqslant \frac{2-2 \sqrt{2 / 3}}{\sqrt{6}-1} .
$$

Case 2.2. Assume $h_{1}>h_{2}$. Thus $y=h_{1}+\epsilon$, and we have

$$
\begin{aligned}
\frac{C_{A L G}\left(S_{5}\right)}{C_{O P T}\left(S_{5}\right)} & =\frac{4+5 h_{1}+3 h_{2}+4 \epsilon}{2+3 h_{1}+h_{2}+3 \epsilon} \\
& \geqslant \frac{4+5 h_{1}+4 \epsilon}{2+3 h_{1}+3 \varepsilon} \quad \text { the function is decreasing w.r.t. } h_{1} \text { for } \epsilon \leqslant 2 / 3 \\
& \geqslant \frac{4+5(\sqrt{2 / 3}-\delta)+4 \epsilon}{2+3(\sqrt{2 / 3}-\delta)+3 \epsilon} \quad \text { as } h_{1} \leqslant \sqrt{\frac{2}{3}}-\delta \\
& \geqslant 1+\sqrt{\frac{2}{3}} \text { let } \epsilon \leqslant \frac{\delta(\sqrt{6}-2)}{\sqrt{6}-1} .
\end{aligned}
$$

In conclusion, we prove that any on-line algorithm has the competitive ratio at least $1+\sqrt{2 / 3}$ in scheduling the adversary job sequence $S$.

By the above lemma, we have actually proved the following theorem that no on-line algorithm can achieve a competitive ratio less than $1+\sqrt{2 / 3}$ in scheduling parallel jobs on two machines.

Theorem 2. For the problem of scheduling parallel jobs on two machines, no on-line algorithm is better than $(1+$ $\sqrt{2 / 3}$-competitive. 


\section{Upper bounds on two special cases}

In this section we study the problem with special case inputs. Section 3.1 considers the job sequences with jobs arriving in a non-decreasing order of processing times. Section 3.2 considers the job sequences with jobs arriving in a non-increasing order of processing times. In these two sections, we analyse the performance of a Greedy Algorithm (defined below) and prove that its competitive ratios are 3/2 and 4/3, respectively. In Section 3.2 we also show that if the jobs arrive in a non-increasing order of processing times, no on-line algorithm can achieve a competitive ratio less than $9 / 7$.

In the following, we define how the Greedy Algorithm schedules a job.

- If the job is $(1, p)$, i.e., a 1-machine job with processing time $p$, then schedule the job at the earliest time $t$ to a machine where the machine is idle throughout the period from time $t$ to $t+p$. If both machines satisfy the requirement for the same earliest time $t$, then schedule the job to Machine 1 at time $t$.

- If the job is $(2, p)$, i.e., a 2-machine job with the processing time $p$, then schedule the job at the earliest time $t$ when both machines are idle throughout the period from time $t$ to $t+p$.

We observe some properties of the greedy schedule. First, there is no time instance in the schedule (from time 0 to the makespan of the schedule) with both machines being idle. Without loss of generality, we can assume that the last job finished in the greedy schedule is a 1-machine job, which is the worst case scenario. Otherwise, removing that job from the input sequence results in a greedy schedule having a larger competitive ratio.

We need some definitions for further discussion. An idle slot $x$ of a machine in the greedy schedule is defined to be a period where the machine is idle and the machine is scheduled to run some jobs immediate before (except for the first idle slot which may start at time 0) and after the period. In fact the job scheduled immediate after the idle slot must be a 2-machine job, otherwise, the job can start earlier which contradicts to the Greedy Algorithm. Let $x_{i}$ denote the $i$-th idle slot of the machines in the greedy schedule ordered by the start time of the slot. For simplicity, $x_{i}$ also denotes the length of the idle slot.

\subsection{Jobs arrive in non-decreasing order of processing times}

In this section we show that the Greedy Algorithm is (3/2)-competitive if the jobs arrive in a non-decreasing order of processing times. In fact, Greedy Algorithm is optimal because no on-line algorithm can achieve a competitive ratio smaller than $3 / 2$ as proved by Faigle et al. [10].

Theorem 3. Greedy Algorithm is (3/2)-competitive in scheduling parallel jobs on two machines if the jobs arrive in a non-decreasing order of processing times.

Proof. In the greedy schedule, for every idle slot $x_{i}$, let $y_{i}$ denote both the 2-machine job and its processing time that starts immediate after $x_{i}$. Let $\ell$ denote both the last (1-machine) job to finish in the greedy schedule and its processing time. Suppose there are $k$ idle slots in the greedy schedule. Let $X=\sum_{i=1}^{k} x_{i}$ be the total length of the idle slots and $Y=\sum_{i=1}^{k} y_{i}$ be the total processing time of the 2-machine jobs starting immediate after the idle slots. Let $C_{\text {Greedy }}$ denote the makespan of the greedy schedule.

Define the total weighted processing time to be the sum of the weighted processing time of all jobs where a $w$ machine job has weight $w$, for $w=1$ or 2 . Note that any schedule has makespan at least half of the total weighted processing time. By the greedy schedule, the total weighted processing time is at least $2 C_{G r e e d y}-X-\ell$. Let $C_{O P T}$ denote the makespan of the optimal schedule. We have $C_{O P T} \geqslant C_{\text {Greedy }}-(X+\ell) / 2$. From another point of view, any schedule must have the 1-machine and 2-machine jobs processed in different time slots, in particular consider the jobs $y_{i}$ and the last job $\ell$. Thus the makespan of any schedule, including the optimal schedule, must be at least $Y+\ell$. As a result, we have $C_{O P T} \geqslant \max \left(C_{\text {Greedy }}-(X+\ell) / 2, Y+\ell\right)$.

We show that $C_{O P T} \geqslant \max \left(C_{\text {Greedy }}-(X+\ell) / 2, Y+\ell\right) \geqslant(2 / 3) C_{\text {Greedy }}$ by considering the following two cases.

(1) Assume $(X+\ell) / 2 \leqslant C_{\text {Greedy }} / 3$. We have $C_{O P T} \geqslant C_{\text {Greedy }}-(X+\ell) / 2 \geqslant(2 / 3) C_{\text {Greedy }}$. 
(2) Assume $(X+\ell) / 2>C_{\text {Greedy }} / 3$. We have $\ell>(2 / 3) C_{\text {Greed }}-X \geqslant(2 / 3) C_{\text {Greed }}-Y$ because each job $y_{i}$ arrives after $x_{i}$ which implies the processing time $y_{i} \geqslant x_{i}$. Therefore $C_{O P T} \geqslant Y+\ell>Y+(2 / 3) C_{\text {Greedy }}-Y=$ $(2 / 3) C_{\text {Greedy }}$.

As a result, we have in any case $C_{O P T} \geqslant(2 / 3) C_{\text {Greedy }}$, i.e., the competitive ratio of the Greedy Algorithm is at most $3 / 2$.

\subsection{Jobs arrive in non-increasing order of processing times}

In this section we show that the Greedy Algorithm is (4/3)-competitive if the jobs arrive in a non-increasing order of processing times and we give a job sequence to illustrate that the analysis is tight. Moreover, we also show that no on-line algorithm can achieve a competitive ratio less than 9/7.

Firstly, we assume that the last job to finish in the greedy schedule does not start at time 0, otherwise, both the greedy and the optimal schedules have the same makespan. For subsequent discussion, we define the all-busy slot in the greedy schedule to be a period where both machines are processing some jobs and either of the two machines is idle immediate before and immediate after the period. Note that for ease of explanation we consider both machines are idle before time 0 and after the makespan. Let $z_{i}$ denote the $i$ th all-busy slot ordered by the start time of the slot. For simplicity, $z_{i}$ also denotes the length of the slot. The following lemma reveals the arrangement of the all-busy slots and the idle slots. It also implies that the number of all-busy slots is one more than the number of idle slots. See Fig. 3 for an example of a greedy schedule.

Lemma 4. Every idle slot is immediately preceded and immediately followed by an all-busy slot in the greedy schedule for jobs arriving in a non-increasing order of processing times.

Proof. Since the greedy schedule does not have any time instance that both machines are idle, every idle slot must be immediately preceded by an all-busy slot, perhaps except the first idle slot which may start at time 0 . We argue that if the last job to finish in the greedy schedule is a 1-machine job (which is assumed), then $x_{1}$ must be preceded by an all-busy slot. Since the first job arrived is the longest job in this special input, which is scheduled at time 0 by the Greedy Algorithm, $x_{1}$ must be at least the value of this processing time. Thus slot $x_{1}$ must be able to accommodate any subsequent jobs including the last job, which implies that an all-busy slot must exist preceding $x_{1}$. The definition of idle slot implies that the slot must be followed by an all-busy slot.

In addition to the idle slots $x_{i}$ defined before, we define the "open" idle period as follows. Suppose Machine 1 and Machine 2 finish all their jobs at time $t_{1}$ and $t_{2}$, respectively, and without loss of generality, let $t_{1} \geqslant t_{2}$. The period from time $t_{2}$ to $t_{1}$, for which Machine 2 is idle, is called the open idle period $x^{\prime}$. For simplicity, $x^{\prime}$ also denotes the length of the period, i.e., $t_{1}-t_{2}$. Similar to the idle slot, the open idle period should be preceded by an all-busy slot, which is the last all-busy slot. Otherwise, the last job must start at time 0 which contradicts to the assumption we made in the beginning of the section. We show the relationship among the all-busy slots, the idles slots, and the open idle period, in the following lemma.

Lemma 5. Suppose the greedy schedule has $k$ idle slots $x_{i}$ and $k+1$ all-busy slots $z_{i}$ for $k \geqslant 0$, and one open idle period. We have $z_{i} \geqslant x_{i}$ for $1 \leqslant i \leqslant k$ and $z_{k+1} \geqslant x^{\prime}$.

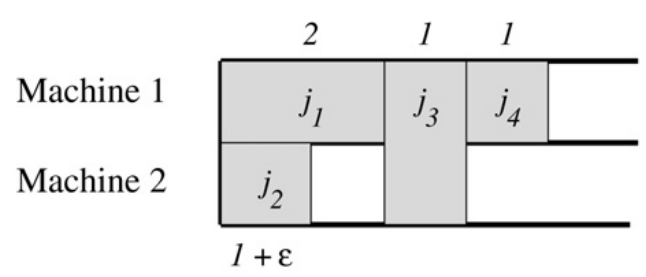

Greedy schedule

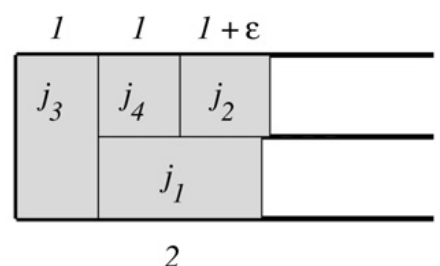

Optimal schedule

Fig. 3. An example to show that greedy schedule has a competitive ratio arbitrarily close to $4 / 3$. 
Proof. We first prove that $z_{i} \geqslant x_{i}$. Consider the slot $x_{i}$ and for instance suppose it appears in Machine 1. We can see that Machine 2 must be processing a single 1-machine job, say $j$, throughout the period of slot $x_{i}$. Otherwise, the second job processed by Machine 2 during slot $x_{i}$ can be scheduled by the Greedy Algorithm to start earlier in Machine 1 during slot $x_{i}$. Then we have the processing time of $j, p_{j} \geqslant x_{i}$. If there is any 2-machine job scheduled in the all-busy slot $z_{i}$, the processing time of that job, say $p^{\prime}$, must be at least $p_{j}$ because the 2-machine job must arrive before job $j$. Hence, we have $z_{i} \geqslant x_{i}$. If there is no 2-machine job scheduled in the all-busy slot $z_{i}, x_{i}$ must be the first idle slot, i.e., $i=1$. There must be a 1-machine job scheduled and completed in slot $z_{1}$ before slot $x_{1}$ in Machine 1 and the processing time of this job is at least $x_{1}$. It is because this job must have the same or longer processing time than that of the last job which cannot be scheduled in slot $x_{1}$. Thus we have proved that $z_{i} \geqslant x_{i}$. In a similar approach, we can also prove $z_{k+1} \geqslant x^{\prime}$.

Theorem 6. Greedy Algorithm is (4/3)-competitive in scheduling parallel jobs on two machines if the jobs arrive in a non-increasing order of processing times.

Proof. Let $X=\sum_{i=1}^{k} x_{i}$ and $Z=\sum_{i=1}^{k+1} z_{i}$. By Lemma 5, we have $Z \geqslant X+x^{\prime}$. Since the greedy schedule does not have any time instance that both machines are idle, we have the makespan of the greedy schedule $C_{\text {Greed }}=Z+X+x^{\prime}$. Since $Z \geqslant X+x^{\prime}$, we also have $Z \geqslant C_{\text {Greedy }} / 2$. On the other hand, the makespan of the optimal schedule $C_{O P T}$ must be at least half the sum of the weighted processing time of all jobs, where the weights of the 1-machine and 2-machine jobs are 1 and 2 , respectively. Therefore, we have $C_{O P T} \geqslant Z+\left(X+x^{\prime}\right) / 2=C_{\text {Greedy }} / 2+Z / 2 \geqslant(3 / 4) C_{\text {Greedy }}$. Hence, the competitive ratio of the Greedy Algorithm is at most 4/3.

We show that the analysis for Theorem 6 is tight by giving a job sequence such that the competitive ratio of the Greedy Algorithm is arbitrarily close to $4 / 3$. Consider a sequence of 4 jobs arriving in the order: $j_{1}=(1,2)$, $j_{2}=(1,1+\epsilon), j_{3}=(2,1)$ and $j_{4}=(1,1)$, where $\epsilon$ is a sufficiently small positive number. The makespan of the greedy schedule is 4 , while the makespan of the optimal schedule is $3+\epsilon$ (see Fig. 3). Thus the competitive ratio is arbitrarily close to $4 / 3$.

In fact, the performance of the Greedy Algorithm is not too bad when compared with the optimal algorithm. In the following theorem, we show that no on-line algorithm is better than $(9 / 7)$-competitive.

Theorem 7. For the problem of scheduling parallel jobs on two machines with the jobs arriving in a non-increasing order of processing times, no on-line algorithm is better than (9/7)-competitive.

Proof. We give an adversary job sequence to show that no on-line algorithm has a competitive ratio less than $9 / 7-\delta$ for any $\delta>0$. The adversary consists of at most 4 jobs, $j_{1}, j_{2}, j_{3}$ and $j_{4}$ arriving one by one in that order. After each job is scheduled, if the competitive ratio of the on-line schedule is at least $9 / 7-\delta$, the adversary stops. We show that if an on-line algorithm has a competitive ratio less than $9 / 7-\delta$ both after scheduling $j_{1}$ and after scheduling $j_{2}$, then the on-line algorithm must have a competitive ratio at least $9 / 7-\delta$ after scheduling all 4 jobs.

Let $j_{1}=(1,1)$. The on-line algorithm must schedule $j_{1}$ at time $h<2 / 7-\delta$, otherwise, the competitive ratio is at least $9 / 7-\delta$. Then let $j_{2}=(2,3 / 4+\epsilon)$ where $\epsilon$ is a sufficiently small positive number. Job $j_{2}$ must be scheduled after $j_{1}$ and at time $1+x$ where $x<1 / 2+2 \epsilon / 7$. Otherwise, the competitive ratio is at least $9 / 7-\delta$. Let $j_{3}=$ $(1,(1+x) / 2+6 \epsilon / 7)$ and $j_{4}=(1,(1+x) / 2+6 \epsilon / 7)$. It can be verified that $(1+x) / 2+6 \epsilon / 7 \leqslant 3 / 4+\epsilon$ because $x<1 / 2+2 \epsilon / 7$. Thus the processing times of all these 4 jobs are in a non-increasing order. Since the sum of the processing times of $j_{3}$ and $j_{4}$ are greater than the length of the idle slot before $j_{2}$, which is $1+x$, at least one of the two jobs must be scheduled after $j_{2}$ (see Fig. 4). Therefore, the competitive ratio of the on-line algorithm after scheduling all 4 jobs is at least

$$
\begin{aligned}
\frac{9 / 4+3 x / 2+13 \epsilon / 7}{7 / 4+x+19 \epsilon / 7} & \geqslant \frac{9 / 4-7 \delta / 4+3 x / 2++7 \delta / 4+13 \epsilon / 7}{7 / 4+x+19 \epsilon / 7} \\
& \geqslant \min \left(9 / 7-\delta, 3 / 2, \frac{7 \delta / 4+13 \epsilon / 7}{19 \epsilon / 7}\right) \\
& =9 / 7-\delta \quad\{\text { let } \epsilon \leqslant 343 \delta / 320\} .
\end{aligned}
$$




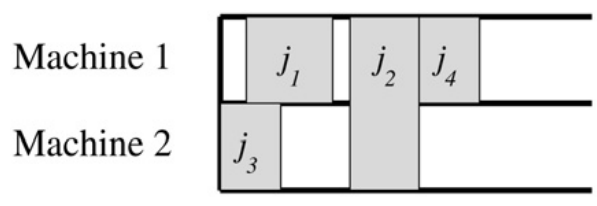

on-line schedule

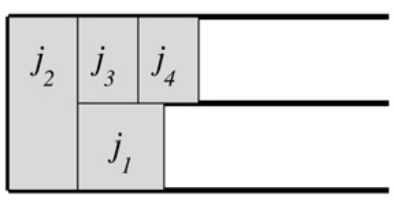

offline schedule

Fig. 4. The on-line and offline schedules for the adversary in the proof of Theorem 7.

By the above argument, if there is an on-line algorithm with a competitive ratio $k<9 / 7$, we can show that the algorithm is at least $(9 / 7-\delta)$-competitive for $9 / 7-\delta>k$, by letting $\delta<9 / 7-k$, which is a contradiction. As a result, we have proved that no on-line algorithm is better than (9/7)-competitive.

\section{References}

[1] S. Bischof, E.W. Mayr, On-line scheduling of parallel jobs with runtime restrictions, Theor. Comput. Sci. 268 (1) (2001) 67-90.

[2] M. Drozdowski, Scheduling parallel tasks—algorithms and complexity, in: J.Y-T. Leung (Ed.), Handbook of Scheduling: Algorithms, Models, and Performance Analysis, CRC Press, Boca Raton, FL, 2004 (Chapter 25).

[3] A. Feldmann, M.-Y. Kao, J. Sgall, S.-H. Teng, Optimal on-line scheduling of parallel jobs with dependencies, J. Comb. Optim. 1 (4) (1998) $393-411$.

[4] E. Naroska, U. Schwiegelshohn, On an on-line scheduling problem for parallel jobs, Inf. Process. Lett. 81 (6) (2002) $297-304$.

[5] D.G. Feitelson, L. Rudolph, U. Schwiegelshohn, Parallel job scheduling—a status report, in: Proceedings of the 10th International Workshop on Job Scheduling Strategies for Parallel Processing (JSSPP 2004), in: Lecture Notes in Computer Science, vol. 3277, Springer, Berlin, 2004, pp. 1-16.

[6] D. Ye, G. Zhang, On-line scheduling of parallel jobs, in: Proceedings of the 11th International Colloquium of Structural Information and Communication Complexity (SIROCCO 2004), in: Lecture Notes in Computer Science, vol. 3104, Springer, Berlin, 2004, pp. $279-290$.

[7] B. Johannes, Scheduling parallel jobs to minimize makespan, Tech. Rep. 723-2001, Technische Universität Berlin, Germany, http://www.math.tu-berlin.de/coga/publications/techreports/2001/Report-723-2001.html, 2001.

[8] A. Borodin, R. El-Yaniv, Online Computation and Competitive Analysis, Cambridge University Press, Cambridge, 1998.

[9] R. Graham, Bounds for certain multiprocessing anomalies, Bell Syst. Tech. J. 45 (1966) 1563-1581.

[10] U. Faigle, W. Kern, G. Turán, On the performance of on-line algorithms for partition problems, Acta Cybern. 9 (2) (1989) $107-119$.

[11] D. Ye, On-line and efficient algorithms for scheduling problems in communication networks, PhD thesis, Zhejiang University, Hangzhou, China, 2005. 